\title{
Giant phonon-induced conductance in scanning tunnelling spectroscopy of gate-tunable graphene
}

\author{
YUANBO ZHANG ${ }^{1 *}$, VICTOR W. BRAR ${ }^{1,2}$, FENG WANG ${ }^{1}$, CAGLAR GIRIT $^{1,2}$, YOSSI YAYON $^{1}$, \\ MELISSA PANLASIGUI ${ }^{1}$, ALEX ZETTL ${ }^{1,2}$ AND MICHAEL F. CROMMIE ${ }^{1,2 *}$ \\ ${ }^{1}$ Department of Physics, University of California at Berkeley, Berkeley, California 94720, USA \\ ${ }^{2}$ Materials Sciences Division, Lawrence Berkeley Laboratory, Berkeley, California 94720, USA \\ *e-mail: zhyb@berkeley.edu; crommie@berkeley.edu
}

The honeycomb lattice of graphene is a unique two-dimensional system where the quantum mechanics of electrons is equivalent to that of relativistic Dirac fermions ${ }^{1,2}$. Novel nanometre-scale behaviour in this material, including electronic scattering ${ }^{3,4}$, spin-based phenomena ${ }^{5}$ and collective excitations ${ }^{6}$, is predicted to be sensitive to charge-carrier density. To probe local, carrier-density-dependent properties in graphene, we have carried out atomically resolved scanning tunnelling spectroscopy measurements on mechanically cleaved graphene flake devices equipped with tunable back-gate electrodes. We observe an unexpected gap-like feature in the graphene tunnelling spectrum that remains pinned to the Fermi level $\left(E_{\mathrm{F}}\right)$ regardless of graphene electron density. This gap is found to arise from a suppression of electronic tunnelling to graphene states near $E_{\mathrm{F}}$ and a simultaneous giant enhancement of electronic tunnelling at higher energies due to a phonon-mediated inelastic channel. Phonons thus act as a 'floodgate' that controls the flow of tunnelling electrons in graphene. This work reveals important new tunnelling processes in gate-tunable graphitic layers.

Graphene provides an ideal platform for the local study of high-mobility two-dimensional (2D) electrons because it can be fabricated on top of an insulating substrate. The availability of a back-gate electrode makes graphene the first gate-tunable 2D system directly accessible to scanning probe measurement (Fig. 1a). Previous experiments have demonstrated the power of scanning tunnelling microscopy (STM) to probe the local electronic structure of graphene grown epitaxially on $\mathrm{SiC}$ (refs 7-9). That system, however, cannot be easily gated, and questions remain as to the influence of the $\mathrm{SiC}$ substrate on the graphene layer ${ }^{6,10}$. Mechanically cleaved graphene is a desirable alternative to graphene grown on SiC because it can be readily gated and placed on wellcontrolled substrates (Fig. 1a), thus making it useful for extracting intrinsic graphene properties.

The STM topography of a gated graphene flake device is shown in Fig. 2a. Corrugations with a lateral dimension of a few nanometres and a vertical dimension of $\sim 1.5 \AA$ (r.m.s. value over a $60 \times 60 \mathrm{~nm}^{2}$ area) are observed, probably due to roughness in the underlying $\mathrm{SiO}_{2}$ (refs 11,12). The graphene honeycomb lattice can be clearly resolved on top of the surface corrugation, as seen more clearly in Fig. 2b.
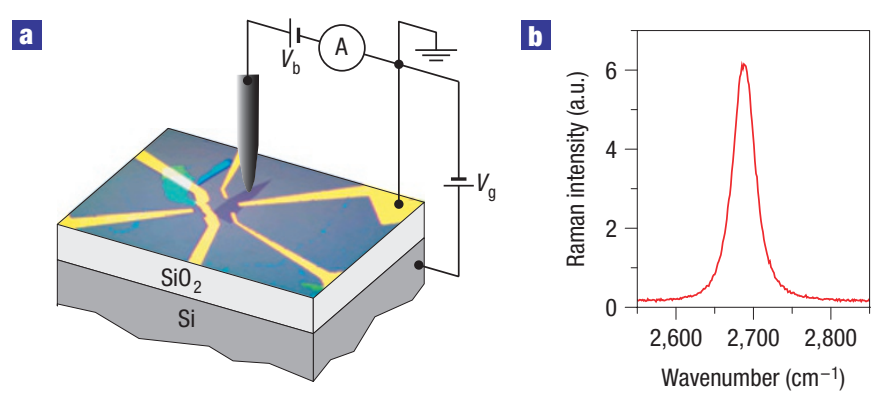

Figure 1 Local probe geometry of gated graphene flake device. a, Optical image of a mechanically cleaved gated graphene flake (dark triangle in centre) accessed by STM tip (tip is sketched). The graphene is contacted by gold electrodes and a back-gate voltage $\left(V_{g}\right)$ is applied to the underlying Si substrate. b, Raman spectrum of the graphene sample in a showing a single peak at wavenumber $\sim 2,700 \mathrm{~cm}^{-1}$, a clear signature for monolayer graphene.

We explored the local electronic structure of these graphene flake devices using $\mathrm{d} I / \mathrm{d} V$ measurements at zero gate voltage, as shown in Fig. 2c. Strikingly, the spectrum shows a $\sim 130 \mathrm{mV}$ gap-like feature centred at the Fermi energy, $E_{\mathrm{F}}$, as opposed to the linear density of states that might be expected from elastic tunnelling to a Dirac cone. A local minimum in the tunnelling conductance spectrum can also be seen at $V_{\mathrm{D}}=-138 \mathrm{mV}$, making the spectrum asymmetric about $E_{\mathrm{F}}$. Close examination of the low-bias spectrum (Fig. 2c, inset) reveals that the tunnelling conductance does not go to absolute zero in the gap region. These observations were reproduced on more than eight different flake devices, and the gap feature was observed in every spectrum acquired with calibrated STM tips (more than 30 different tips). The gap feature was seen to be independent of location on a sample, although the energy position of the adjacent dip feature, $V_{\mathrm{D}}$, varied with tip location. This observation (together with the fact that a similar gap feature of the same size was seen on a graphene/SiC sample ${ }^{9}$ ) is evidence against substrate-induced effects as the origin of the gap feature, because such effects would 
a

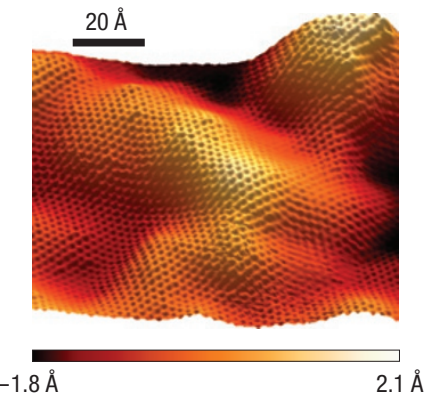

b

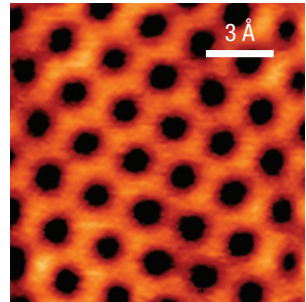

$-0.1 \AA$

$0.1 \AA$

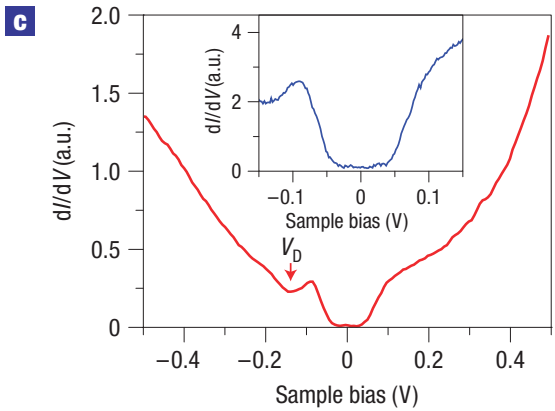

Figure 2 Graphene surface topography and differential conductance $(\mathrm{d} I / \mathrm{d} V)$ spectrum. a, Constant-current STM topograph ( $1 \mathrm{~V}, 50 \mathrm{pA})$ of a graphene flake above a $\mathrm{Si}_{2}$ substrate. b, Close-up constant-current STM topograph $(0.15 \mathrm{~V}, 40 \mathrm{pA})$ of the graphene honeycomb lattice. $\mathbf{c}, \mathrm{d} / / \mathrm{d} V$ spectrum of graphene at zero gate voltage. The spectrum was acquired at a nominal junction impedance of $5 \mathrm{G} \Omega$ $(0.5 \mathrm{~V}, 100 \mathrm{pA})$. The gap width and the adjacent conductance minimum location (at $V_{D}$ ) were not sensitive to STM tip height over an impedance range of 1-100 G $\Omega$. Inset: A high-resolution $\mathrm{d} / / \mathrm{d} V$ spectrum emphasizing the central gap-like feature.

imply a gap that depends on spatial location as well as the type of substrate.

Changing the device gate voltage, $V_{\mathrm{g}}$, causes the graphene Dirac point $\left(E_{\mathrm{D}}\right)$ to shift energetically relative to $E_{\mathrm{F}}$, inducing a $2 \mathrm{D}$ charge-carrier density of $n=\alpha V_{\mathrm{g}}$, where $\alpha=7.1 \times 10^{10} \mathrm{~cm}^{-2} \mathrm{~V}^{-1}$ (estimated using a simple capacitor model ${ }^{1,2}$ ). STM $\mathrm{d} I / \mathrm{d} V$ spectra taken at the same location on graphene with gate voltages ranging from $V_{\mathrm{g}}=-60$ to $+60 \mathrm{~V}$ are shown in Fig. $3 \mathrm{a}$. The width and energy position of the central gap feature do not show any dependence on gate voltage, but the conductance minimum at $V_{\mathrm{D}}$ shifts monotonically with gate voltage, and even switches polarity (red arrows, Fig. 3a).

To further characterize the mysterious central gap feature, we measured both the temperature dependence and the effective barrier for electrons tunnelling into graphene. As shown in Fig. 4a, no significant temperature dependence is observed in graphene $\mathrm{d} I / \mathrm{d} V$ spectra measured at $T=4.8$ and $77.5 \mathrm{~K}$ (this lack of temperature dependence was observed for $-60 \mathrm{~V}<V_{\mathrm{g}}<+60 \mathrm{~V}$ ). Tunnel barrier measurements were carried out by measuring the STM tunnel current as a function of tip-sample separation $(z)$ at constant bias for voltages inside and outside the gap, and identical measurements were also carried out on a gold sample using the same tip as a calibration. STM tunnel current depends exponentially on $z, I \propto \mathrm{e}^{-z / \lambda}$, and the decay length $(\lambda)$ gives a measure of the effective tunnel barrier. $\lambda$ was obtained by fitting $I$ versus $z$ measurements at large $z$ (tunnel junction resistance $>30 \mathrm{G} \Omega$ ) with an exponential function. As seen in Fig. $4 \mathrm{~b}, \lambda$ for graphene is comparable to that observed in the gold calibration data $(\lambda \approx 0.45 \AA)$ for biases outside the gap, but
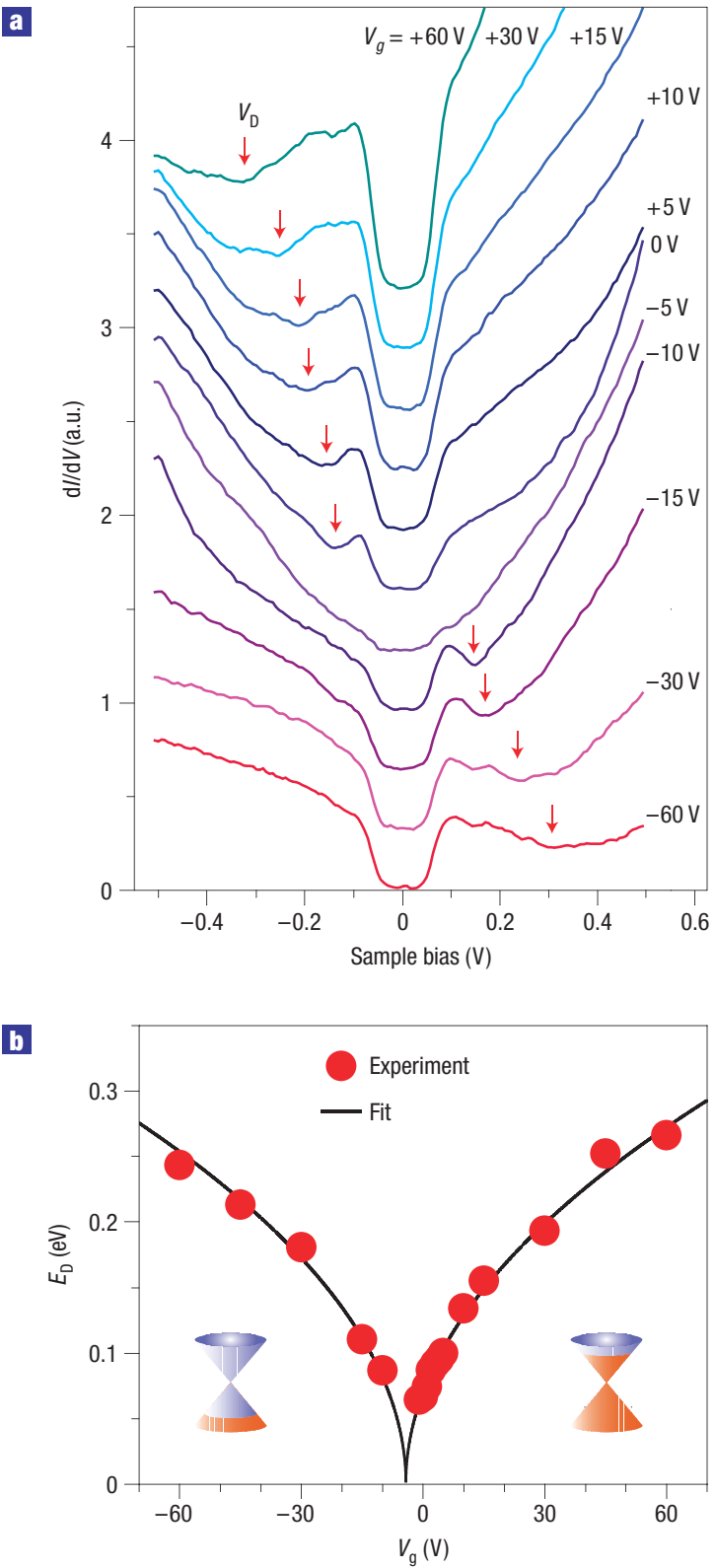

Figure 3 Gate-voltage dependence of graphene tunnelling spectra. $a, d / / d V$ spectra taken at the same point on the graphene surface for different gate voltages, $V_{\mathrm{g}}$. Spectra are acquired at the same nominal junction impedance, $5 \mathrm{G} \Omega(0.5 \mathrm{~V}$, $100 \mathrm{pA}$ ). Curves are vertically displaced for clarity. Red arrows indicate the gate-dependent positions of the adjacent conductance minimum, $V_{D}$, outside the gap feature. $\mathbf{b}$, Energy position of the Dirac point, $E_{\mathrm{D}}$, as a function of applied gate voltage (extracted from the conductance minimum in a using equation (1): $\left.E_{\mathrm{D}}=e\left|V_{\mathrm{D}}\right|-\hbar \omega_{0}\right)$. $E_{\mathrm{D}}$ and its associated uncertainty (which is smaller than the size of the symbols) are obtained by fitting the minimum with polynomials. The black curve is a fit to the data using the square-root dependence of $E_{\mathrm{D}}$ on gate voltage. The inset schematic diagrams show linear graphene energy bands when a gate voltage is applied (filled states are orange).

decreases markedly to a value only half as large $(\lambda \approx 0.25 \AA)$ for biases within the gap.

How do we interpret the anomalous graphene energy gap behaviour and gate-voltage-dependent conductance minima? A consistent picture emerges if we consider phonon-mediated 

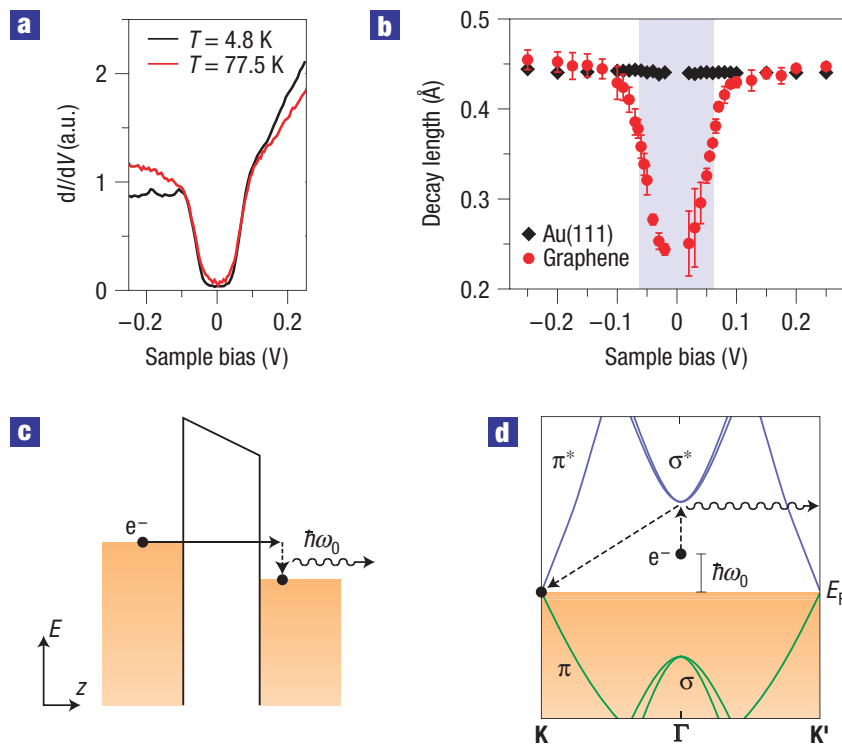

Figure 4 Characterization of the Fermi level gap feature and inelastic electron tunnelling mechanism. a, Graphene $\mathrm{d} / \mathrm{d} V$ conductance spectra measured at $T=4.8 \mathrm{~K}$ and $77.5 \mathrm{~K}$ (no significant temperature dependence is observed). b. Tunnel current decay length of graphene and $\mathrm{Au}(111)$ surfaces probed at different bias voltages with the same STM tip. Error bars represent standard deviations of the measurements. c, Inelastic electron tunnelling due to excitation with energy $\hbar \omega_{0}$. d, Wave-vector-dependent inelastic tunnelling mechanism involving graphene phonon modes near the $\mathbf{K}$ point in reciprocal space.

inelastic tunnelling of electrons into the graphene flake accompanied by a strong suppression of elastic tunnelling at $E_{\mathrm{F}}$. In what follows, we first establish a general inelastic origin of the central gap feature and we then discuss how our data can be explained by a novel phonon-based inelastic excitation mechanism.

STM tunnel current is generally enhanced if the bias voltage is high enough to provide tunnelling electrons with enough energy to induce excitations that have some threshold energy $\hbar \omega_{0}$ (Fig. 4c) ${ }^{13}$. This opens up a new inelastic tunnelling channel at bias voltages of $\pm \hbar \omega_{0} / e$, causing steps in $\mathrm{d} I / \mathrm{d} V$ spectra that are symmetric around $E_{\mathrm{F}}$ and that lead to a gap-like feature with width $2 \hbar \omega_{0}$. In the case of our graphene measurements, $\hbar \omega_{0}=63 \pm 2 \mathrm{meV}$ (this corresponds to one half of the voltage separation between peak centres in $\left.\mathrm{d}^{2} I / \mathrm{d} V^{2}\right)$. Strong evidence for such an inelastic tunnelling mechanism in graphene (as opposed to some other gapinducing mechanism) can be seen in our data by analysing the gatevoltage dependence of the conductance minimum observed at $V_{\mathrm{D}}$. If we assume that this minimum arises from inelastic tunnelling to the graphene Dirac point (a minimum in the density of states), then its energy location in our data $\left(e V_{\mathrm{D}}\right)$ should be offset by $\hbar \omega_{0}$ from its true energy location, $E_{\mathrm{D}}$, in the graphene band structure (because each inelastically tunnelling electron loses energy $\hbar \omega_{0}$ ):

$$
E_{\mathrm{D}}=e\left|V_{\mathrm{D}}\right|-\hbar \omega_{0}
$$

Because $V_{\mathrm{D}}$ depends on gate voltage, this inelastic relation enables us to directly plot $E_{\mathrm{D}}$ versus $V_{\mathrm{g}}$, as shown in Fig. $3 \mathrm{~b}$. Identification of the conductance minimum at $V_{\mathrm{D}}$ with the Dirac point energy, $E_{\mathrm{D}}$, can be confirmed by fitting this plot with the expected dependence $E_{\mathrm{D}}=\hbar v_{\mathrm{F}} \sqrt{\pi \alpha\left|V_{\mathrm{g}}-V_{0}\right|}$ as prescribed by the graphene linear band structure ${ }^{1,2}$. Here, $v_{\mathrm{F}}$ is the Fermi velocity of graphene and $V_{0}$ is the shift of the Dirac point (in terms of gate voltage) due to substrate doping. An excellent fit is obtained with $v_{\mathrm{F}}=1.10 \pm 0.01 \times 10^{6} \mathrm{~m} \mathrm{~s}^{-1}$ and $V_{0}=-4.1 \pm 0.2 \mathrm{~V}$, values that are consistent with those of previous studies ${ }^{1,2,14}$. This unambiguously identifies the observed conductance minimum at $V_{\mathrm{D}}$ as the Dirac point and simultaneously verifies a graphene inelastic tunnelling channel as the origin of the central gap feature.

The question next arises as to what specific type of graphene inelastic excitation we are observing that has energy $\hbar \omega_{0} \approx 63 \mathrm{meV}$. One possible attribution is the $67 \mathrm{meV}$ out-of-plane acoustic graphene phonon modes located near the $\mathbf{K} / \mathbf{K}^{\prime}$ points in reciprocal space $^{15}$. Electrons with energy less than this phonon threshold energy tunnel elastically into graphene at $E_{\mathrm{F}}$ (near the $\mathbf{K}$ point) with only a low probability due to suppression of electronic tunnelling into states with a large wave vector ${ }^{16,17}$. Once the threshold bias voltage $\pm \hbar \omega_{0} / e$ is reached, however, tunnelling into $\mathbf{K}$ point states is markedly enhanced (seen as more than a factor of 10 increase in tunnelling conductance) by the opening of a new inelastic channel. In this new mechanism, an electron first tunnels into the graphene $\sigma^{*}$ band near the $\boldsymbol{\Gamma}$ point in reciprocal space (a virtual transition as shown in Fig. $4 \mathrm{~d}$ ) before falling into an available $\mathbf{K}$ point state on the $\pi$ band through the emission of a $\mathbf{K}^{\prime}$ point out-of-plane phonon (to conserve crystal momentum and energy). This phonon-mediated inelastic tunnelling process, which involves momentum-conserving virtual transitions between $2 \mathrm{D}$ electronic bands, is distinctly different from previously studied inelastic tunnelling in single molecules ${ }^{13,18}$ or localized spins ${ }^{19}$ where momentum is not a well-defined quantity owing to a lack of translational symmetry (phonon-induced inelastic tunnelling in single molecules typically leads to conductivity changes of the order of only $1 \%$ in contrast to the factor of 10 seen here ${ }^{18}$ ). This is also different from band-structure-dependent tunnelling in silicon ${ }^{20}$ because the wave-vector dependence seen here is a result of inelastic excitations that couple the unique electronic band structure and phonon spectrum of graphene. A recent calculation $^{21}$ by Wehling et al. confirms this general interpretation through density functional theory. They find significant mixing of the graphene $\sigma$ and $\pi$ electron bands due to the out-ofplane phonon mode at $\mathbf{K} / \mathbf{K}^{\prime}$, resulting in a strongly enhanced inelastic electron tunnel current. We note that there are three in-plane phonon modes at $\mathbf{K} / \mathbf{K}^{\prime}$, but their contributions to this process are insignificant owing to the symmetries of the $\sigma$ and $\pi$ bands $^{22}$.

This mechanism is strongly supported by our observed wavefunction spatial decay rates. Within the observed energy gap (at energies below the inelastic threshold), electrons have to tunnel directly into graphene states with large crystal momentum parallel to the surface $\left(\boldsymbol{k}_{\|}=\mathbf{K}\right.$ or $\left.\mathbf{K}^{\prime}\right)$. Such states tend to decay rapidly in the vacuum region above a surface, because their evanescent local density of states is expected to fall off as ${ }^{16}$

$$
\operatorname{LDOS}\left(z, k_{\|}\right) \propto \mathrm{e}^{-z / \lambda}, \quad \lambda^{-1}=2 \sqrt{2 m \phi / \hbar^{2}+k_{\|}^{2}},
$$

where $\phi$ is the workfunction and $m$ is the mass of an electron. This accounts for the strong suppression of tunnelling conductance within the energy gap region and leads to the small decay length observed in the low-bias elastic tunnelling channel. At voltages outside the gap (at energies above the inelastic threshold), however, electrons tunnel via virtual excitations to the $\sigma^{*}$-band states near the $\Gamma$ point which have $k_{\|} \approx 0$. Such states tend to extend further into the vacuum, leading to a larger decay length. Using the two measured decay lengths $\left(\sim 0.45 \AA\right.$ for $k_{\|}=0$ and $\sim 0.25 \AA$ for $k_{\|}=K$ ) combined with equation (2), we are able to extract a workfunction of $\phi=4.6 \pm 0.2 \mathrm{eV}$ and a graphene $\mathrm{K}$ point wave vector $K=1.7 \pm 0.4 \AA^{-1}$. These values agree well with the known workfunction of graphite and the known graphene 
K point wavevector $K=1.7 \AA^{-1}$. This behaviour explains why the gap feature seen here is not observed by other experimental techniques (such as angle-resolved photoemission spectroscopy and transport measurements) that are not sensitive to the spatial decay of the electron wavefunction at a surface. The observed lack of temperature dependence in our $\mathrm{d} I / \mathrm{d} V$ spectra can also be explained by the fact that the $\mathbf{K} / \mathbf{K}^{\prime}$ phonon energy is so much higher than $k_{\mathrm{B}} T$ ( $k_{\mathrm{B}}$ is the Boltzmann constant).

It is possible to gain insight into the electron-phonon coupling strength that underlies the inelastic tunnelling process observed here. The ratio of phonon-mediated tunnelling conductance at energies just outside the gap compared with elastic tunnel conductance at energies inside the gap can be approximated as follows (see the Supplementary Information):

$$
\left(\frac{\mathrm{d} I}{\mathrm{~d} V}\right)_{\text {out }} /\left(\frac{\mathrm{d} I}{\mathrm{~d} V}\right)_{\text {in }} \approx \frac{1}{7.4}\left(\frac{V_{\mathrm{el}-\mathrm{ph}}}{E_{\sigma}}\right)^{2} \exp \left[\left(\lambda_{k_{\|}=0}^{-1}-\lambda_{k_{\|}=K}^{-1}\right) z\right] .
$$

Here, $V_{\mathrm{e}-\mathrm{ph}}$ is the intrinsic graphene electron-phonon coupling matrix element connecting electronic states at the $\boldsymbol{\Gamma}$ and $\mathbf{K}$ points in reciprocal space and $E_{\sigma}$ is the energy of intermediate states near the $\Gamma$ point on the $\sigma^{*}$ band $\left(E_{\sigma} \sim 4 \mathrm{eV}\right.$; ref. 23$)$. If we take the STM tip height as $z \approx 5 \AA$ (estimated from the tip-sample junction impedance $\approx 2 \mathrm{G} \Omega$ ) and use our measured ratio of $(\mathrm{d} I / \mathrm{d} V)_{\text {out }} /(\mathrm{d} I / \mathrm{d} V)_{\text {in }} \approx 13$, then equation (3) enables us to extract an experimental electron-phonon coupling strength $V_{\mathrm{el}-\mathrm{ph}} \approx 0.4 \mathrm{eV}$, consistent with a theoretical estimate of $V_{\mathrm{el}-\mathrm{ph}} \approx 0.5 \mathrm{eV}$ based on a simple tight-binding model ${ }^{22}$ (see equations 5.16, 5.18 and 5.20 and figure 5-9 in ref. 24). Our experimental value of $V_{\mathrm{el}-\mathrm{ph}}$, however, should only be taken as a very rough estimate because the absolute tip-height above graphene, $z$, is not directly measured.

In summary, we have demonstrated the ability to measure atomically resolved local electronic structure of a graphene flake device while changing its charge-carrier density using a back gate. The resulting graphene spectra exhibit a prominent gap-like feature that arises from a unique phonon-mediated inelastic tunnelling process that is intrinsic to graphene. Phonons thus serve as a 'floodgate' in graphene by promoting electronic tunnelling above a phonon threshold energy. The resulting nonlinear tunnelling $I-V$ behaviour will impact future graphene device applications that use electron tunnelling processes.

\section{METHODS}

Our graphene monolayers were prepared in a similar fashion as in ref. 25 . Monolayers of graphene were identified using an optical microscope, and were subsequently confirmed using Raman spectroscopy (Fig. 1b) ${ }^{26,27}$. Gold electrodes $(30 \mathrm{~nm})$ were attached to graphene using direct deposition through a stencil mask for surface cleanliness. The gold contacts remained Ohmic up to a source-drain voltage of $500 \mathrm{mV}$ (contact resistance $<300 \Omega$ ). Heavily doped $\mathrm{Si}$ under the $\mathrm{SiO}_{2}$ layer was used as a back gate, enabling us to vary the carrier density in the graphene (Fig. 1a). All graphene samples were annealed at $400^{\circ} \mathrm{C}$ in ultrahigh vacuum for $\sim 10 \mathrm{~h}$ to ensure surface cleanliness before STM measurements.

Our STM measurements were carried out in an Omicron low-temperature STM at $T=4.8 \mathrm{~K}$ and in an ultrahigh-vacuum environment with base pressure $<10^{-11}$ mbar. STM measurements were conducted with chemically etched metal STM tips made of tungsten or platinum/iridium alloy. To ensure that our STM tips were free of anomalies in their electronic structure, we calibrated the tips by carrying out tunnelling differential conductance $(\mathrm{d} I / \mathrm{d} V)$ measurements on a clean $\mathrm{Au}(111)$ surface both before and after graphene measurement. This calibration is crucial for obtaining reproducible intrinsic tunnelling spectra on graphene because tip contamination is known to induce spurious artefacts ${ }^{28}$ (see the Supplementary Information). $\mathrm{d} I / \mathrm{d} V$ spectra were measured through lock-in detection ${ }^{9}$.

\section{Received 6 February 2008; accepted 5 June 2008; published 20 July 2008.}

\section{References}

1. Novoselov, K. S. et al. Two-dimensional gas of massless Dirac fermions in graphene. Nature $\mathbf{4 3 8}$ 197-200 (2005).

2. Zhang, Y., Tan, Y.-W., Stormer, H. L. \& Kim, P. Experimental observation of the quantum Hall effect and Berry's phase in graphene. Nature 438, 201-204 (2005).

3. Katsnelson, M. I., Novoselov, K. S. \& Geim, A. K. Chiral tunnelling and the Klein paradox in graphene. Nature Phys. 2, 620-625 (2006).

4. Cheianov, V. V., Fal'ko, V. \& Altshuler, B. L. The focusing of electron flow and a Veselago lens in graphene p-n junctions. Science 315, 1252-1255 (2007).

5. Son, Y. W., Cohen, M. L. \& Louie, S. G. Half-metallic graphene nanoribbons. Nature 444, 347-349 (2006).

6. Bostwick, A., Ohta, T., Seyller, T., Horn, K. \& Rotenberg, E. Quasiparticle dynamics in graphene. Nature Phys. 3, 36-40 (2007)

7. Rutter, G. M. et al. Scattering and interference in epitaxial graphene. Science 317, 219-222 (2007)

8. Mallet, P. et al. Electron states of mono- and bilayer graphene on $\mathrm{SiC}$ probed by scanning-tunneling microscopy. Phys. Rev. B 76, 041403 (2007).

9. Brar, V. W. et al. Scanning tunneling spectroscopy of inhomogeneous electronic structure in monolayer and bilayer graphene on SiC. Appl. Phys. Lett. 91, 122102 (2007).

10. Zhou, S. Y. et al. Substrate-induced bandgap opening in epitaxial graphene. Nature Mater. 6 , 770-775 (2007).

11. Ishigami, M., Chen, J. H., Cullen, W. G., Fuhrer, M. S. \& Williams, E. D. Atomic structure of graphene on $\mathrm{SiO}_{2}$. Nano Lett. 7, 1643-1648 (2007).

12. Stolyarova, E. et al. High-resolution scanning tunneling microscopy imaging of mesoscopic graphene sheets on an insulating surface. Proc. Natl Acad. Sci. 104, 9209-9212 (2007).

13. Lambe, J. \& Jaklevic, R. C. Molecular vibration spectra by inelastic electron tunneling. Phys. Rev. 165 821 (1968).

14. Martin, J. et al. Observation of electron-hole puddles in graphene using a scanning single electron transistor. Nature Phys. 4, 144-148 (2008).

15. Mohr, M. et al. Phonon dispersion of graphite by inelastic x-ray scattering. Phys. Rev. B 76, 035439 (2007).

16. Tersoff, J. \& Hamann, D. R. Theory and application for the scanning tunneling microscope. Phys. Rev. Lett. 50, 1998-2001 (1983).

17. Vitali, L. et al. Phonon and plasmon excitation in inelastic electron tunneling spectroscopy of graphite. Phys. Rev. B 69, 121414(R) (2004).

18. Stipe, B. C., Rezaei, M. A. \& Ho, W. Single-molecule vibrational spectroscopy and microscopy. Science 280, 1732-1735 (1998).

19. Hirjibehedin, C. F., Lutz, C. P. \& Heinrich, A. J. Spin coupling in engineered atomic structures. Science 312, 1021-1024 (2006)

20. Stroscio, J. A. Feenstra, R. M. \& Fein, A. P. Electronic-structure of the Si(111) $2 \times 1$ surface by scanning-tunneling microscopy. Phys. Rev. Lett. 57, 2579-2582 (1986).

21. Wehling, T. O., Grigorenko, I., Lichtenstein, A. I. \& Balatsky, A. V. Phonon mediated tunneling into graphene. Preprint at $<$ http://arxiv.org/abs/0803.4427> (2008).

22. Jiang, J. et al. Electron-phonon matrix elements in single-wall carbon nanotubes. Phys. Rev. B 72 , 235408 (2005).

23. Sugawara, K., Sato, T., Souma, S., Takahashi, T. \& Suematsu, H. Fermi surface and edge-localized states in graphite studied by high-resolution angle-resolved photoemission spectroscopy. Phys. Rev. B 73, 045124 (2006).

24. Samsonidze, G. PhD thesis (Massachusetts Institute of Technology, Massachusetts, 2006).

25. Novoselov, K. S. et al. Two-dimensional atomic crystals. Proc. Natl Acad. Sci. 102 10451-10453 (2005).

26. Ferrari, A. C. et al. Raman spectrum of graphene and graphene layers. Phys. Rev. Lett. 97, 187401 (2006).

27. Gupta, A., Chen, G., Joshi, P., Tadigadapa, S. \& Eklund, P. C. Raman scattering from high-frequency phonons in supported n-graphene layer films. Nano Lett. 6, 2667-2673 (2006).

28. Tiedje, T., Varon, J., Deckman, H. \& Stokes, J. Tip contamination effects in ambient pressure scanning tunneling microscopy imaging of graphite. J. Vac. Sci. Technol. A 6, 372-375 (1988).

Supplementary Information accompanies this paper on www.nature.com/naturephysics.

Acknowledgements

We thank D.-H. Lee, S. Louie, A. Balatsky, J. Moore, C. H. Park, G. Samsonidze, D. Wegner, L. Berbil-Bautista and C. Hirjibehedin for helpful discussions. This work was supported by the DOE under contract No. DE-AC03-76SF0098. Y.Z. and F.W. acknowledge postdoctoral fellowships and A.Z. acknowledges a professor fellowship, from the Miller Institute, UC Berkeley.

\section{Author information}

Reprints and permission information is available online at http://npg.nature.com/reprintsandpermissions Correspondence and requests for materials should be addressed to Y.Z. or M.F.C. 